\begin{tabular}{|c|c|c|c|}
\hline av Sor & Daniel Benhuri, M & Ujj & $\mathbf{R a}$ \\
\hline $\begin{array}{l}\text { Department of Cardiovascular Medicine, } \\
\text { Mount Sinai Beth Israel, } \\
\text { New York, NY }\end{array}$ & $\begin{array}{l}\text { Department of Cardiovascular Medicine, } \\
\text { Mount Sinai Beth Israel, } \\
\text { New York, NY }\end{array}$ & $\begin{array}{l}\text { Department of Cardiovascular Medicine, } \\
\text { Mount Sinai Beth Israel, } \\
\text { New York, NY }\end{array}$ & $\begin{array}{l}\text { Department of Cardiovascular Medicine, } \\
\text { Mount Sinai Beth Israel, } \\
\text { New York, NY }\end{array}$ \\
\hline
\end{tabular}

\title{
Pacemaker lead-induced venous thoracic outlet syndrome
}

\begin{abstract}
A 73-YEAR-OLD WOMAN came to our ofA fice. She had chronic nonvalvular atrial fibrillation, hypertension, systemic lupus erythematosus (in remission), and sick sinus syndrome for which a pacemaker had been implanted 2 years ago. She presented with acute onset of left arm swelling and pain after having taken a domestic airline flight and missing a single dose of her direct oral anticoagulant. She presented to us approximately 10 days after symptom onset.

Examination revealed a blood pressure of 120/78 mm Hg, heart rate 88 beats per minute, and respiratory rate 14 per minute without any distress. lows:

Results of laboratory testing were as fol-

- Hemoglobin $11.1 \mathrm{~g} / \mathrm{dL}$ (reference range $11.7-15 \mathrm{~g} / \mathrm{dL}$ )

- Activated partial thromboplastin time $41.5 \mathrm{sec}(25-37 \mathrm{sec})$

- International normalized ratio 1.3 (0.91.1)

- Anticardiolipin antibody less than $9 \mathrm{U} / \mathrm{mL}$ (0-11 U/mL).

Duplex ultrasonography of the left upper extremity revealed possible thrombosis or obstruction of the left subclavian vein. She was referred for contrast venography, which revealed a thrombus in the subclavian vein along with venous collaterals (Figure 1).

The patient was admitted for ultrasonography-assisted catheter-guided thrombolysis utilizing alteplase along with unfractionated heparin infusion for 24 hours. The next day, she underwent repeat venography, which revealed marked resolution of the thrombus and
\end{abstract}

doi:10.3949/ccjm.88a.20023
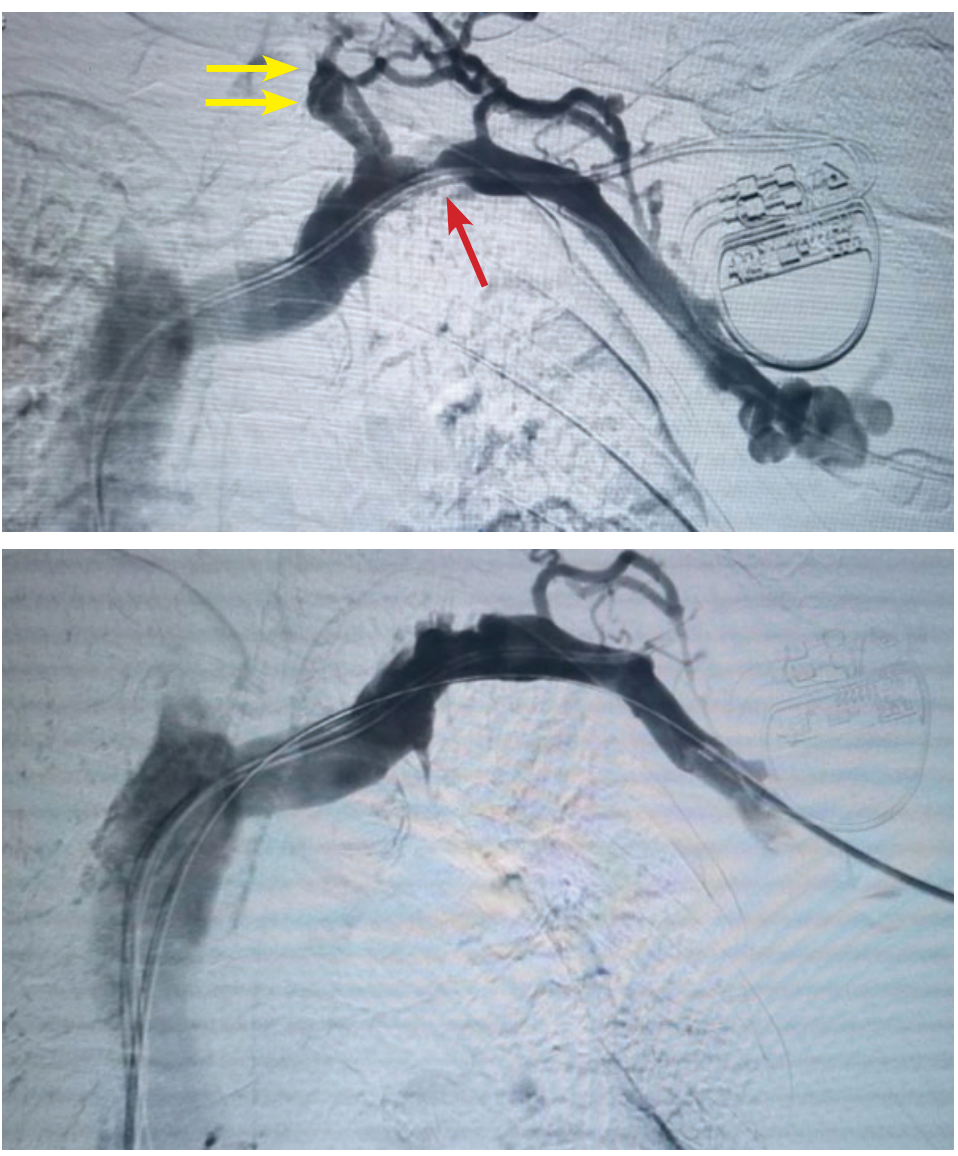

Figure 1. Initial venography (top) depicted a thrombus in the subclavian vein (red arrow) with venous collaterals (yellow arrows). Venography after thrombolysis and angioplasty (bottom) showed reconstitution of the subclavian vein with disappearance of collaterals. Pacemaker leads and catheter are seen in both images.

focal high-grade stenosis of the subclavian vein, which was treated with balloon angioplasty with excellent luminal gain and disappearance of the collaterals.

She continued her oral anticoagulation. 

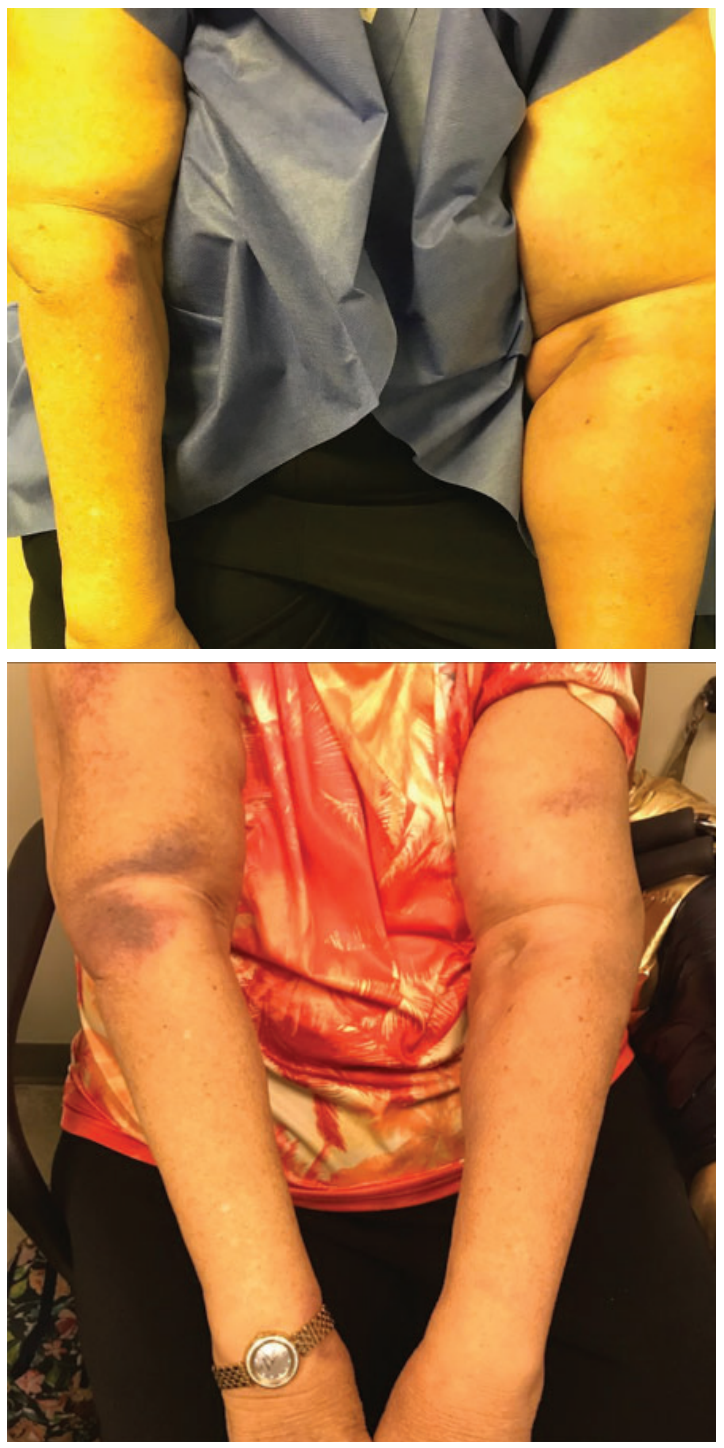

Figure 2. Unilateral left arm swelling at presentation (top) had diminished at 8 weeks after the start of treatment.

At her last follow-up visit, 8 months after her venous stenting, the swelling had diminished substantially (Figure 2), and she remained free of symptoms and signs suggestive of venous stent stenosis or occlusion. She has been maintained on anticoagulation.

\section{VENOUS THORACIC OUTLET SYNDROME}

Venous thoracic outlet syndrome is the second most common subtype of thoracic outlet syndrome after neurologic thoracic outlet syndrome. ${ }^{1}$ It is an uncommon cause of unilateral arm swelling, and needs to be differentiated from deep vein thrombosis involving the brachial or axillary veins.

Venous thoracic outlet syndrome can be primary (from recurrent compression trauma to the subclavian vein from surrounding anatomic structures, leading to thrombosis) or secondary (from pacemaker leads or a hypercoagulable state). ${ }^{2}$ Differentiating the types is important and is based on the patient's history, which influences treatment. Primary venous thoracic outlet syndrome affects young people involved in activities requiring strenuous arm use such as tennis and weightlifting, and commonly affects the dominant arm, as opposed to secondary venous thoracic outlet syndrome, which occurs without a predisposing age or occupation.

\section{APPROACHES TO MANAGEMENT}

Management of venous thoracic outlet syndrome varies in different institutions, as there are no official guidelines. ${ }^{1}$ However, there is a general consensus that primary venous thoracic outlet syndrome, within the first 2 weeks, should be managed by thrombolysis with surgical resection of the first rib. Anticoagulation alone is not the treatment of choice. Angioplasty is indicated in cases with focal venous defects. Secondary venous thoracic outlet syndrome is managed with thrombolysis and anticoagulation, with angioplasty for focal venous disease and removal of the inciting insult when possible, eg, removing the pacemaker lead or changing it to the opposite side.

\section{DISCLOSURES}

The authors report no relevant financial relationships which, in the context of their contributions, could be perceived as a potential conflict of interest.

\section{REFERENCES}

1. Moore R, Wei Lum Y. Venous thoracic outlet syndrome. Vasc Med 2015; 20(2):182-189. doi:10.1177/1358863X14568704

2. Illig KA, Doyle AJ. A comprehensive review of PagetSchroetter syndrome. J Vasc Surg 2010; 51(6):1538-1547. doi:10.1016/j.jvs.2009.12.022

Address: Abhinav Sood, MBBS, MD, Department of Cardiovascular Medicine, Mount Sinai Beth Israel, 281 1st Avenue, New York, NY 10003; abhinavsood56@yahoo.com; abhinav.sood@mountsinai.org 